\title{
Educational Case Study on H-Infinity Robust Performance Design
}

\author{
Jakub NEMCIK, Filip KRUPA, Stepan OZANA, Lukas HUBKA, Zdenek SLANINA
}

\begin{abstract}
This paper deals with the design of a robust controller according to the $\mathrm{H}$-infinity norm of mixed sensitivity functions. The motivation and origin are given by educational reasons. It compares how settings of individual weighting functions affect the quality of control when designing a sub-optimal controller. First, a controller for a system without uncertainty is considered (a nominal system representing a motor with a flexible clutch) and then the design is modified for a perturbated system with uncertainty in gain and also in the time constant.
\end{abstract}

Keywords: $\mathrm{H}$-infinity control; $\mathrm{H}$-infinity optimization; robust control; robust stability; robustness

\section{INTRODUCTION}

In modern control theory, the concept of "robust control" originated in the second half of the $20^{\text {th }}$ century. Robustness can be achieved with single-input/singleoutput (SISO) systems by fulfilling a sufficient phase and gain margin, resulting in a good quality of control. In case of multidimensional systems, a linear quadratic Gaussian method (LQG) was used to design a robust control circuit, see $[1,2]$.

Scientists who devote themselves to control theory have found out how important it is to consider a model of uncertainty in order to achieve a desired quality, which has not been considered in existing optimal feedback control of the multidimensional systems. This lack of optimal control has inspired scientists with a deeper study of the robustness of control systems rather than optimality, resulting in a better performance of design feedback control circuits in terms of eliminating the differences between the system model and the real system, see [4].

Conventional control theories, including some advanced ones, use a system model for the control design of the plant to be controlled. However, this model is never accurate because values of some parameters may remain unknown or may change over time, or there is a mismatch due to neglected nonlinearity or due to unmodelled dynamics. Furthermore, there may be a situation where a simplified model of an originally complex system is intentionally used, or there is no other option than to apply such a simplification. However, in this case the uncertainty only covers a part of dynamics. All the above-mentioned circumstances lead to the introduction of uncertainties into the system model. Instead of a single so-called nominal model, the whole class of so-called perturbed models is defined, which means models in the neighbourhood of the nominal model, i.e. burdened with an uncertainty, see [5].

Robustness means that a certain property provided by a controller for a single nominal system will be also guaranteed for the entire class of perturbed systems. It can also be evaluated for classical control algorithms $(P, I, D)$. Mostly, however, the term robust control is referred to when the uncertainty is taken into account explicitly and systematically when designing the controller.

\section{MATHEMATICAL BACKGROUND}

The design of a robust controller is aimed at finding a controller that would meet the requirement of robust control quality (robust performance) for the entire class of the systems where a nominal plant is just a special sample that belongs to the group. Here is a list of common methods that are used to design a robust controller (see $[2,3,6])$ :

- $H_{\infty}$;

$-\mathrm{H}_{2}$;

- Loop Shaping method;

- Loop Transfer Recovery (LTR);

- $\quad \mu$-synthesis;

- Quantitative Feedback Theory (QFT);

- etc.

The requirements placed on behaviour and robustness of the control circuit can be formulated using maximum amplitudes $M_{\mathrm{S}}=\|S(j \omega)\|_{\infty}$ and $M_{\mathrm{T}}=\|T(j \omega)\|_{\infty}$ where $S$ and $T$ represent a sensitivity, resp. complementary sensitivity function, limited by the basic constraint expressed as $S+T=1$. Typical requirements are as follows: $M_{\mathrm{S}}<2(\equiv 6 \mathrm{~dB})$ and $M_{\mathrm{T}}<1.25$. If the values of $M_{\mathrm{S}}$ and $M_{\mathrm{T}}$ are high, the dynamic performance of the control circuit will be poor and low robustness will occur.

In the ideal case, it is required for $S$ to be small, so it is sufficient to deal only with the $|S|$ amplitude, without considering the phase. Typically, $|S|$ is small at low frequencies; at high frequencies it gets up to one due to strict fineness. At medium frequencies it is often possible to prevent the peak from reaching 1 which indicates that within this area the dynamic behaviour of the control system is getting worse. Limiting peak of $S$, which represents the highest degradation rate, makes it possible to prevent the amplification of a noise at medium and higher frequencies, and it also introduces a degree of robustness.

Mathematically, the requirements can be expressed using the value of upper limit applied for $S$ in the following form:

$$
\frac{1}{\left|W_{\mathrm{P}}(s)\right|}
$$


The weighting function $W_{\mathrm{P}}(s)$ is specified by the user. The requirement on control quality for the nominal plant (nominal performance) can be expressed as follows:

$$
|S(j \omega)|<\frac{1}{W_{\mathrm{P}}(s)} \forall \omega \Leftrightarrow\left|W_{\mathrm{P}}(s) \cdot S(j \omega)\right|<1 \forall \omega
$$

which, using the $H_{\infty}$ norm, results in final form:

$$
\left\|W_{\mathrm{P}}(s) \cdot S(j \omega)\right\|_{\infty}<1
$$

The requirement can be interpreted graphically (see Fig. 1). The distance of an open-loop frequency characteristic in complex plane from the point $(-1 ; 0)$ must be greater than the absolute value of the weight function $W_{\mathrm{P}}(j \omega)$ for all frequencies. The point $L_{0}(j \omega)$ defined at a specific frequency must lie outside the circle centered at $(-1 ; 0)$ having the radius of $W_{\mathrm{P}}(j \omega)$. That guarantees sufficient values of the phase and gain margins.

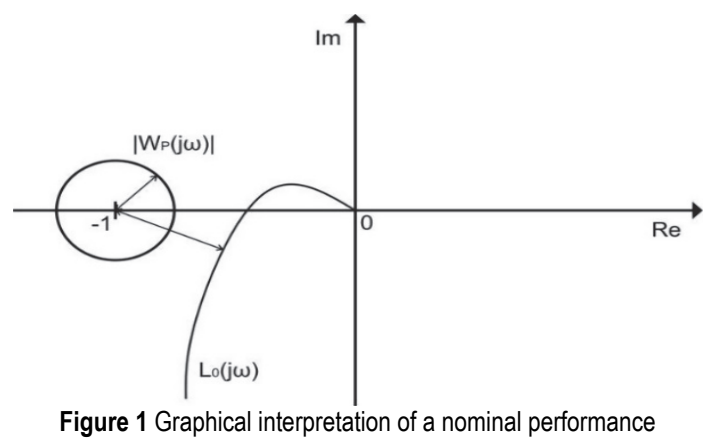

\subsection{Robust Stability}

A robustly stable circuit is one that is stable not only for the nominal system $G_{0}(s)$, but also for all systems in its neighbourhood defined by the model with uncertainty, also referred to as the family. A controller capable of stabilizing both $G_{0}(s)$ and all systems falling under the group $G(s)$ is robustly stabilizing.

Assuming a stable open-loop transfer function $L(s)$ a closed-loop transfer function $T(s)$ is stable if the following holds good:

$\|L(j \omega)\|<1 \forall \omega$

In case of uncertainty, the feedback system is robustly stable when the following holds good:

$$
\left\|W_{\mathrm{M}}(j \omega) \cdot T_{0}(j \omega)\right\|<1
$$

where $W_{\mathrm{M}}$ represents an envelope representing the upper boundary of uncertainty and $T_{0}$ stands for a closed loop of the nominal model.

Similarly, to nominal performance, robust stability can also be expressed graphically, see Fig. 2. The uncertainty of the system must not change the number of cycles of the open-loop frequency characteristic (in the complex plane) of the nominal system $L_{0}(j \omega)$ around the point $(-1 ; 0)$. This means that the Nyquist diagram's envelope must not contain a critical point $(-1 ; 0)$.

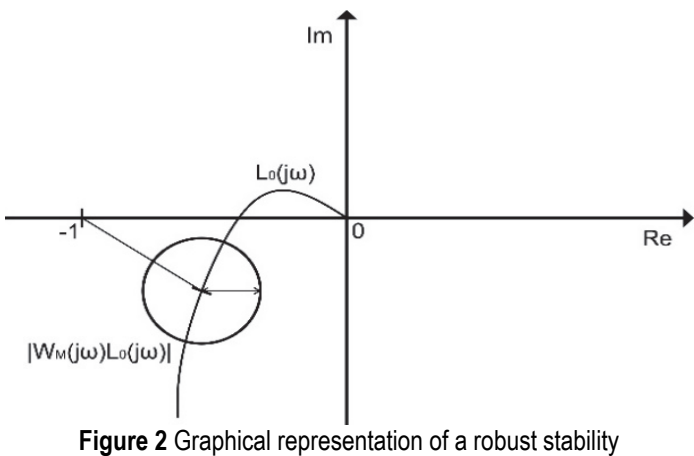

\subsection{Robust Performance}

Robust performance means meeting a robust control quality for the entire system family (i.e., even for perturbed systems, including the worst case). This requirement can be formulated as a combination of nominal performance and robust stability:

$$
\left|W_{\mathrm{P}}(j \omega)\right|+\left|W_{\mathrm{M}}(j \omega) \cdot L_{0}(j \omega)\right|<\left|L_{0}(j \omega)-(-1)\right| \forall \omega s
$$

In terms of graphical interpretation of this requirement, robust performance is achieved if the intersection of the circle centered at $(-1 ; 0)$ having the radius $W_{\mathrm{P}}(j \omega)$ and a circle centered at $L_{0}(j \omega)$ having the radius of $\left|W_{\mathrm{M}}(j \omega) \cdot L_{0}(j \omega)\right|$ is a void.

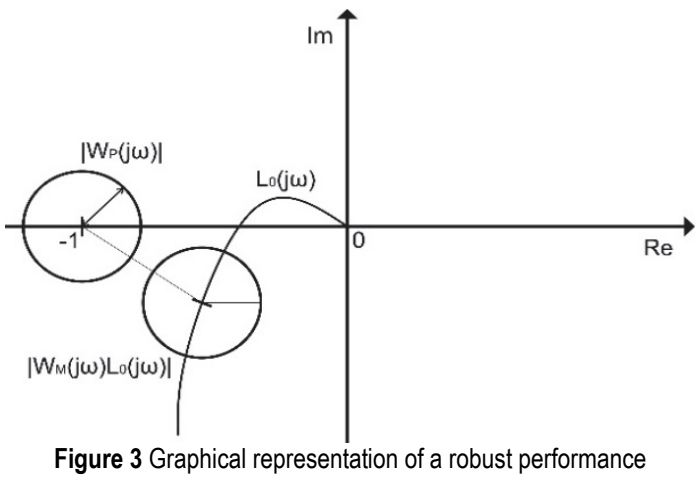

\subsection{Mixed Sensitivity Problem}

As mentioned above, $S$ and $T$ cannot be minimized at all frequencies simultaneously. For this reason, the weighting functions are introduced (see Fig. 4, [7, 8]), so that other variables can be weighed in addition to $S$ and $T$. These penalty functions, or filters, help shape and thus tune to the open- loop and closed-loop behaviour. The individual weighting functions will be described in detail below.

\section{CASE STUDY}

To illustrate the mixed sensitivity problem, a motor speed control with a flexible clutch is considered. Based on identification at the operating point, the following nominal transfer function was obtained:

$$
G_{0}(s)=\frac{0.2964}{0.0257 \cdot s^{3}+0.1781 \cdot s^{2}+0.2991 \cdot s+1}
$$




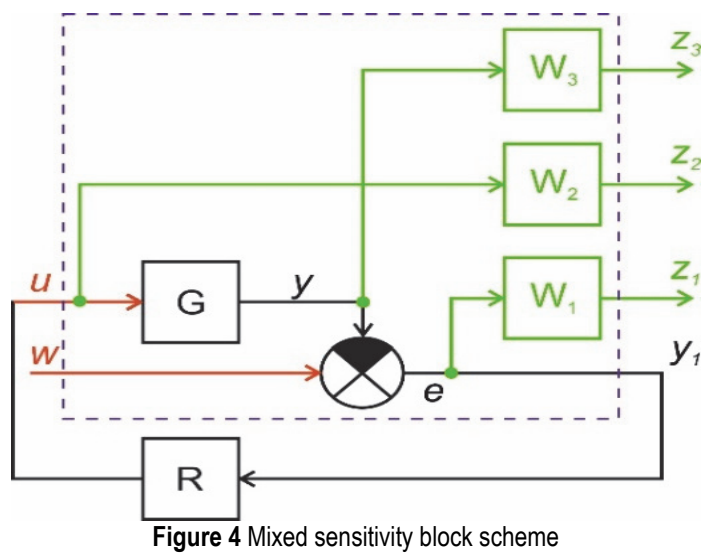

To compare the effect of filter settings, firstly a suboptimal $H_{\infty}$ controller designed to minimize the $H_{\infty}$ norm of the mixed sensitivity is only considered for the nominal system (no uncertainties), i.e. no filter function $W_{3}$ is considered. Thus, a model of the closed-loop control circuit containing only $W_{1}$ and $W_{2}$ penalty filters is obtained, see Fig. 5. The transfer function $W_{1}$ is equal to the transfer function $W_{\mathrm{P}}$ mentioned in the sections related to nominal performance, robust stability, and robust performance.

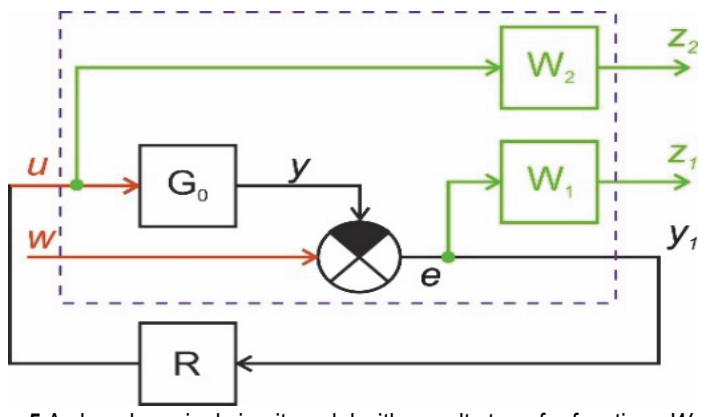

Figure 5 A closed nominal circuit model with penalty transfer functions $W_{1}$ a $W_{2}$

\subsection{Design of $\mathrm{H}_{\infty}$ Controller-Nominal Performance}

The suboptimal controller design itself can be performed in several ways. In this paper, two Matlab functions were used. Namely, it is mixsyn and hinfsynfunctions. The most crucial difference in these functions is mainly the form of entering input parameters for calculation of the controller.

The input parameters of the mixsyn function are the weighting transfer functions together with plant transfer function, whereas the input parameter of the hinfsyn function is the block transfer matrix $\boldsymbol{P}$ (packed matrix) of the generalized closed circuit in the form based on matrices $\boldsymbol{A}, \boldsymbol{B}, \boldsymbol{C}$ and $\boldsymbol{D}$, where for this case the $\boldsymbol{P}$ matrix can be calculated using the function augw. Optionally, it is possible to calculate the matrix $\boldsymbol{P}$ according to the following equation:

$\boldsymbol{P}(s)=\left[\begin{array}{cc}W_{1}-G \cdot W_{1} \\ 0 & W_{2} \\ 1 & -G\end{array}\right]$

In case of the synthesis of the controller according to the $H_{\infty}$-norm of mixed sensitivities it is necessary to know or to choose the penalty transfer functions (filters), and to set them correctly. The choice of penalty transfer means a design of a filter in order to limit appropriate signal. In general, to set the penalty transfer function, it is possible to use one of the basic filter transfer functions described by three parameters as follows:

$W(s)=\frac{\frac{s}{m}+\omega_{\mathrm{B}}}{s+\omega_{\mathrm{B}} \cdot A_{\mathrm{L}}}$

where $A_{\mathrm{L}}$ is the filter gain at low frequencies, the $M$ is the gain at high frequencies, and $\omega_{\mathrm{B}}$ the intersection of frequency characteristic with the $x$-axis (crossoverfrequency).

The filter $W_{1}$, resp. $W_{\mathrm{P}}$, helps to define requirements on the quality of control, because it determines a limitation for a control error. Using the inversion of this transfer function it is possible to define the area (upper boundary) of the amplitude of the sensitivity function $S$.

Accordingly, the following parameters have been selected:

$M=10 ; \omega_{B}=10 \mathrm{rad} \cdot s^{1} ; A_{\mathrm{L}}=0.001$

Using these values with Eq. (9) leads to the penalty transfer function of $W_{1}$ as follows:

$W_{1}(s)=\frac{\frac{s}{10}+10}{s+0.1}=\frac{10 \cdot(s+100)}{(100 \cdot s+1)}$

Requirements placed on manipulated value are set defined by penalty transfer function $W_{2}$. In this case study, it was chosen as follows Eq. (12):

$W_{2}(s)=0.1$

The resulting suboptimal controller for a nominal system representing a motor with a flexible clutch has the $4^{\text {th }}$ order transfer function with a pair of conjugate complex poles and two real poles:

$R_{1}(s)=\frac{19502 \cdot(s+6.065) \cdot\left(s^{2}+0.8611 \cdot s+6.416\right)}{(s+645.8) \cdot(s+0.01) \cdot\left(s^{2}+15.63 \cdot s+104.8\right)}$

This controller minimizes the $H_{\infty}$-norm of the mixed sensitivity functions $W_{1}(s) \cdot S$ and $W_{2}(s) \cdot R \cdot S$ of the closed loop and it is suboptimal because it meets the following requirements:

$$
\left.\begin{array}{c}
\bar{\sigma} \cdot(S(j \omega)) \leq \gamma \cdot \underline{\sigma}\left(W_{1}^{-1}(S(j \omega))\right) \\
\bar{\sigma} \cdot(S \cdot R(j \omega)) \leq \gamma \cdot \underline{\sigma}\left(W_{2}^{-1}(S(j \omega))\right)
\end{array}\right\} \forall \omega
$$

These requirements can also be expressed graphically using singular values, see Fig. 6 which demonstrates fulfilling of the above-mentioned Eq. (14). 


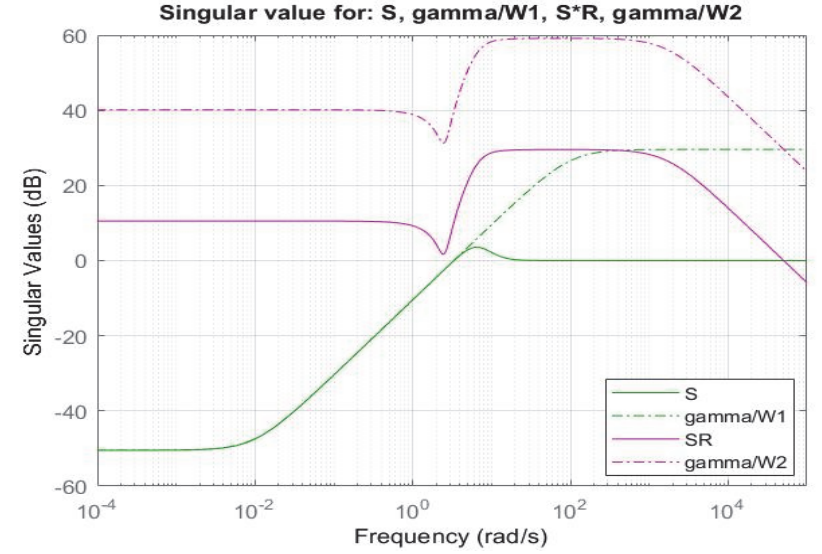

Figure 6 Singular values: graphical representation

\subsection{Influence of Setting of Transfer Functions $W_{1}$ and $W_{2}$ on the Output-Controlled Variable}

An integral part of the control design is also verification of the manipulated value and output value from the system. The choice of parameters of penalty weighting transfer functions may seem complicated, because shaping of the filter in the frequency domain should not only lead to achievement of the desired time domain waveforms, but also to fulfilling the number of additional requirements.

As mentioned above, the penalty transfer function $W_{1}$ serves to achieve the desired quality control. The transfer function includes three parameters that influence the quality of regulation. Increasing of the high frequency gain from the original value $M=10$ up to $M=100$ does not influence the design of the controller for the motor with a flexible clutch almost at all. On the contrary, decreasing of this value $(M=0.1)$ significantly increases the rise time, see Fig. 7.

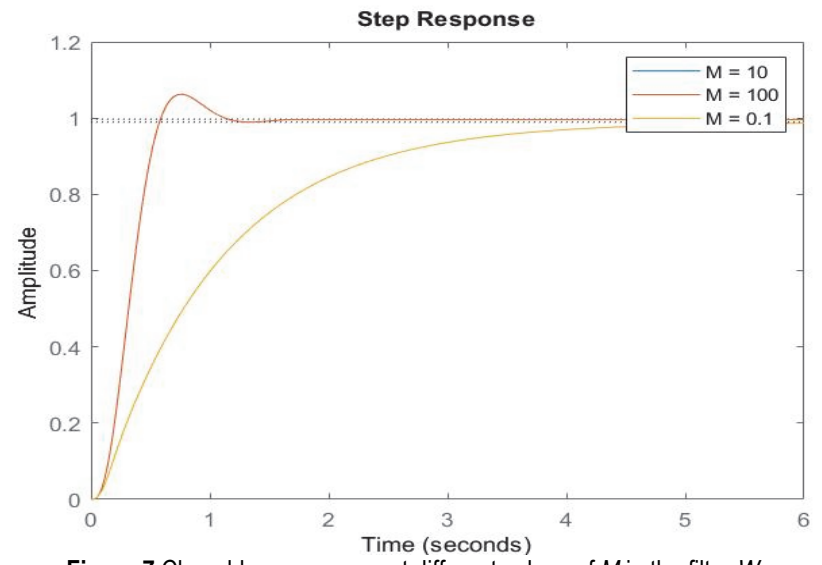

Figure 7 Closed-loop response at different values of $M$ in the filter $W_{1}$

The change of a gain at low frequencies represented by parameter $A_{\mathrm{L}}$ has a significant effect. The higher the value of $A_{\mathrm{L}}$, the smaller the manipulated value, see Fig. 8 and Fig. 9. With the use of parameter $A_{\mathrm{L}}$, it is possible to determine steady-state control error. Interpretation of this parameter can be explained on an example as follows: a decrement of $-20 \mathrm{~dB}$ corresponds to 0.1 of absolute gain, which can be considered as a reasonable admissible tolerance. Considering higher value, such as $10 \%$ for example, is completely pointless. By changing the crossoverfrequency of the penalty transfer function $W_{1}$, resp. by its increase, it is possible to achieve a faster response of the control circuit, see Fig. 10. However, this speedup is attended by a peak increase in manipulated value (see Fig. $11)$, which is impossible to apply in practice.

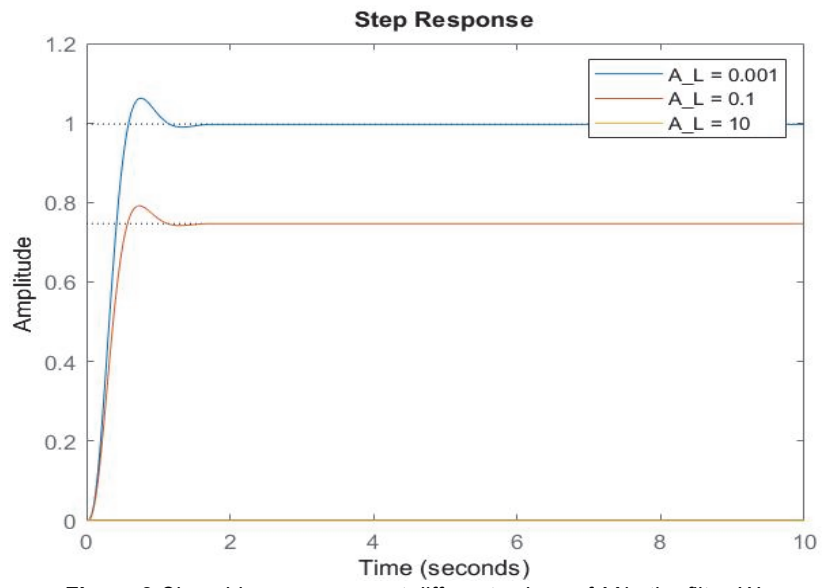

Figure 8 Closed-loop response at different values of $M$ in the filter $W_{1}$

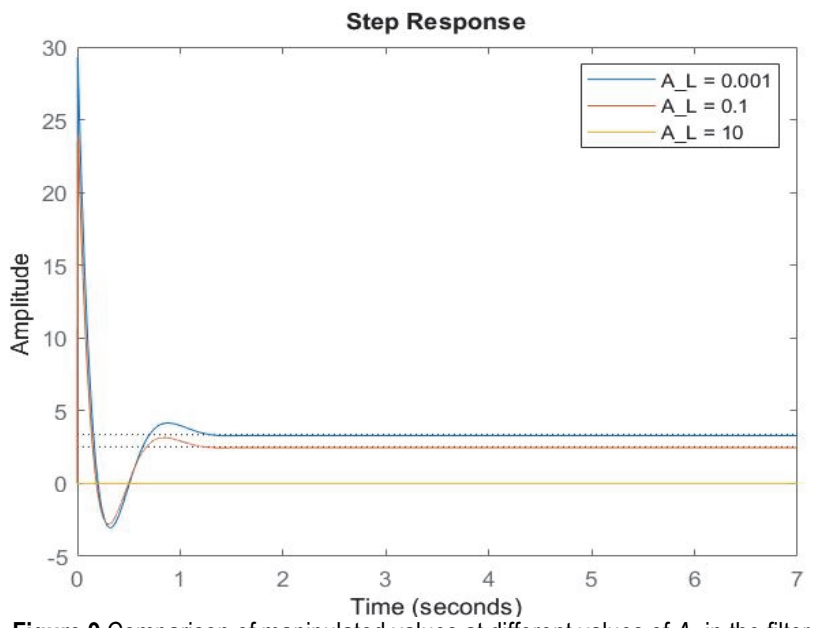

Figure 9 Comparison of manipulated values at different values of $A_{L}$ in the filter $W_{1}$

For this reason, it is possible to limit the manipulated value by $\mathrm{W}_{2}$ filter. Similarly, to $\mathrm{W}_{1}, \mathrm{~W}_{2}$ can also be frequency dependent by which it is possible to suppress the amplitudes of manipulated value and thus restrict "derivative" character (peek) at appropriate frequency. It was initially set to a constant value, indicating a frequency independence. At higher values of $\mathrm{W}_{2}$ values, the peek in the manipulated value is reduced, see Fig. 12.

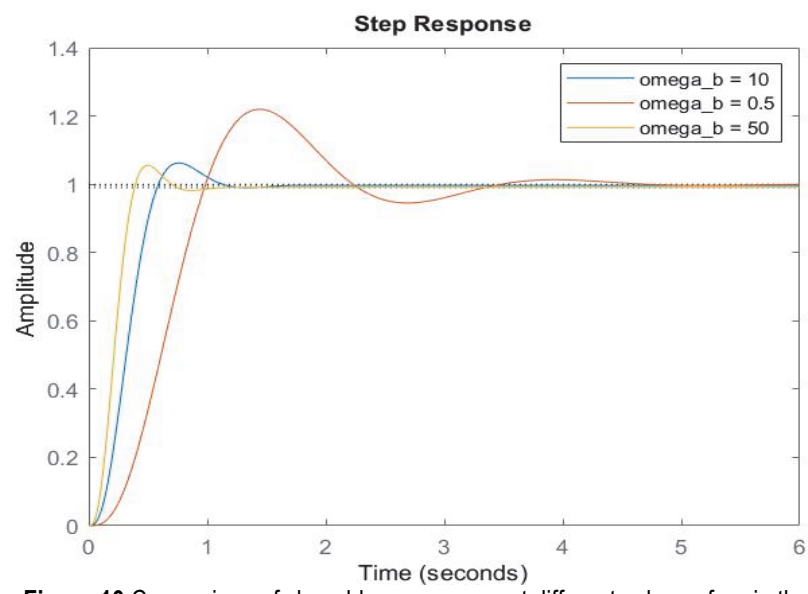

Figure 10 Comparison of closed-loop response at different values of $\omega_{\mathrm{B}}$ in the filter $W_{1}$ 


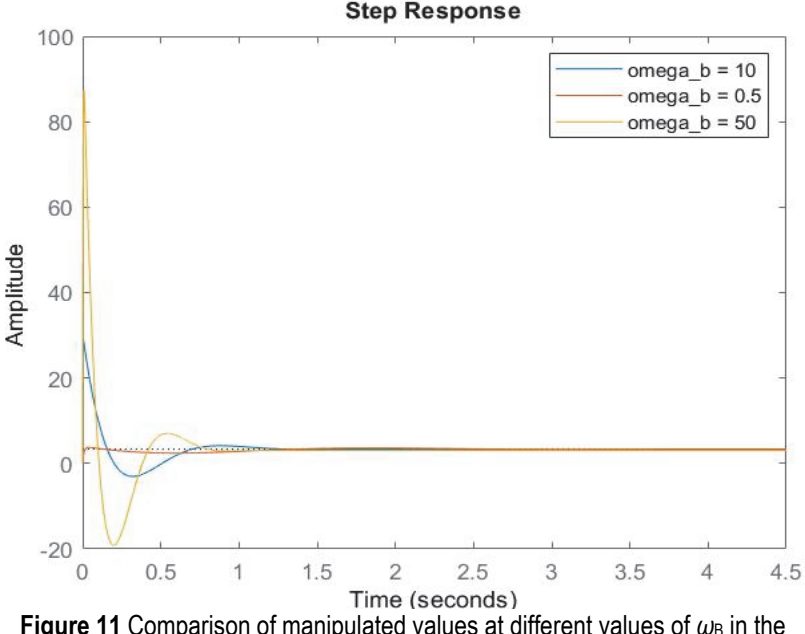

Figure 11 Comparison of manipulated values at different values of $\omega_{B}$ in the filter $W_{1}$

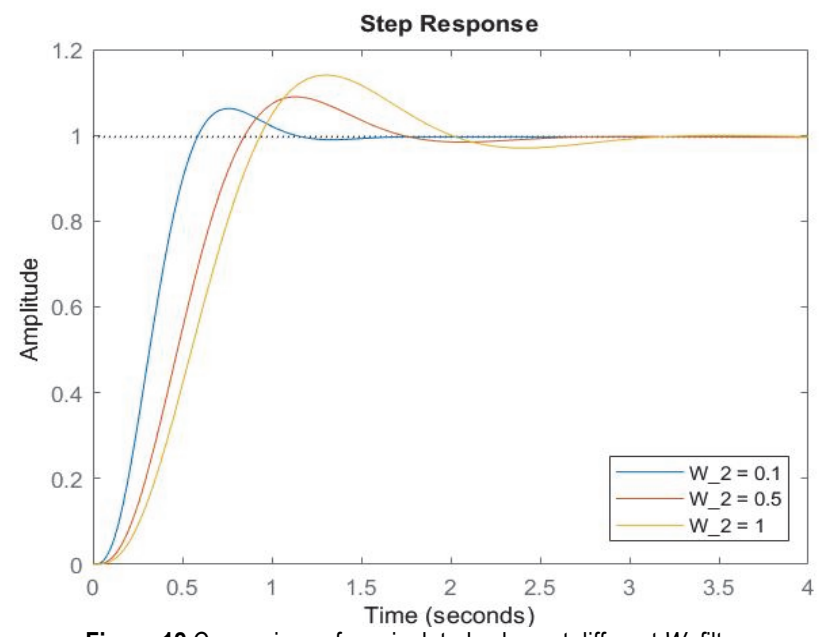

Figure 12 Comparison of manipulated values at different $W_{2}$ filters

\subsection{Introduction of Uncertainty in Gain}

Suppose that the nominal transfer function $G_{0}$ is burdened with an uncertainty in its gain within the range of $\pm 10 \%$, which can be expressed as follows Eq. (15):

$$
k=0.2964 \pm 0.1 \cdot 0.2964 \rightarrow k \in\langle 0.26676 ; 0.32604\rangle
$$

Uncertainty in gain can be introduced into the system using a multiplicative uncertainty model as follows:

$$
G(s)=\left(1+W_{\mathrm{M}}(s) \cdot \Delta_{\mathrm{M}}(s)\right) \cdot G_{0}
$$

where $\Delta_{\mathrm{M}}$ represents the uncertainty itself provided that $\left\|\Delta_{\mathrm{M}}(s)\right\| \leq 1$ and $W_{\mathrm{M}}$ can be expressed by the penalty function $W_{3}$, whereby it is possible to redraw the whole control scheme into the form depicted in Fig. 4. Such a control scheme thus includes three penalty transfer functions, a perturbated plant (plant burdened with an uncertainty in gain) and a controller. The choice of an envelope corresponding to the worst possible case, given by $W_{3}$, is made with the use of plotting the amplitude characteristics of the difference between nominal and perturbed system. Based on this characteristic, the worst case can be characterized by $W_{3}(s)=0.1$ which represents the upper boundary of uncertainty. In case of a motor with a flexible clutch, this penalty transfer function represents $10 \%$ of the gain, thereby using Eq. (16) results in transfer function of the perturbed system in the following form:

$$
G(s)=\left(1+0.1 \cdot \Delta_{\mathrm{M}}(s)\right) \cdot G_{0}=1.1 \cdot \Delta_{\mathrm{M}}(s) \cdot G_{0}
$$

The controller design was performed similarly to the nominal system using MATLAB, yet the penalty function $W_{3}$ has already been introduced in this case, and the resulting controller $R_{2}$ has been designed for a family of perturbed plants. The difference in robustness can be simplified using the Nyquist open-loop diagrams, see Fig. 13. It is obvious that $R_{2}$ controller is much more robust since the Nyquist plot is farther away from the point $(-1$; $0)$.

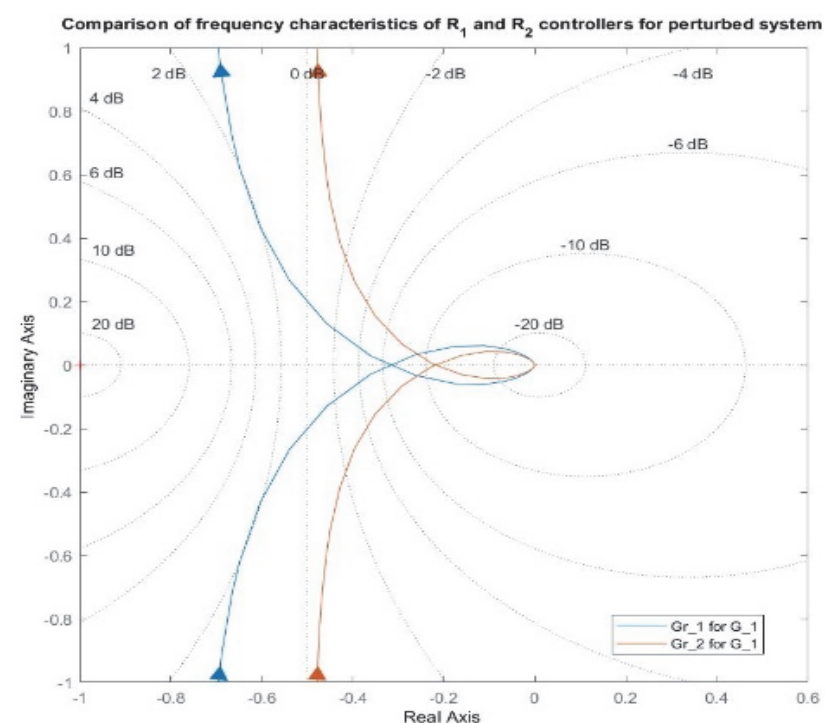

Figure 13 Nyquist open-loop diagrams for particular controllers for nominal and perturbated plant (worst case)

\subsection{Introduction of Uncertainty in Gain and Time Constant}

The procedure for introducing uncertainty in gain and time constant is similar to the previous case. First, however, it is necessary to express the nominal transfer function to the form with time constants and then to introduce the uncertainty correctly into the system. A simple modification of Eq. (7) leads to the following form of $G_{0}$ :

$$
G_{0}=\frac{0.2964}{0.0257 \cdot 6.065 \cdot\left(\frac{1}{6.065} \cdot s+1\right) \cdot\left(s^{2}+0.8611 \cdot s+6.416\right)}
$$

It is supposed that this time the nominal plant $G_{0}$ is not only burdened with a change in the gain, but also in the time constant, within the range of $10 \%$. The change in gain is defined above Eq. (15) and the change in the time constant can be expressed as follows:

$$
T=0.1649 \pm 0.1 \cdot 0.1649 \rightarrow T \in\langle 0.1484 ; 0.1814\rangle
$$

The uncertainty in both the gain and the time constant can again be introduced into the system by a multiplicative 
uncertainty model. The settings for $W_{3}$ filter have been made again by plotting the amplitude characteristic of the difference between the nominal system and all possible perturbed systems, see Fig. 14. The resulting weighting transfer function of $W_{3}$ indicating the upper boundary for uncertainty, which is necessary for the controller design, is supposed as follows:

$W_{3}=\frac{\frac{s}{0.171}+40}{s+4}$

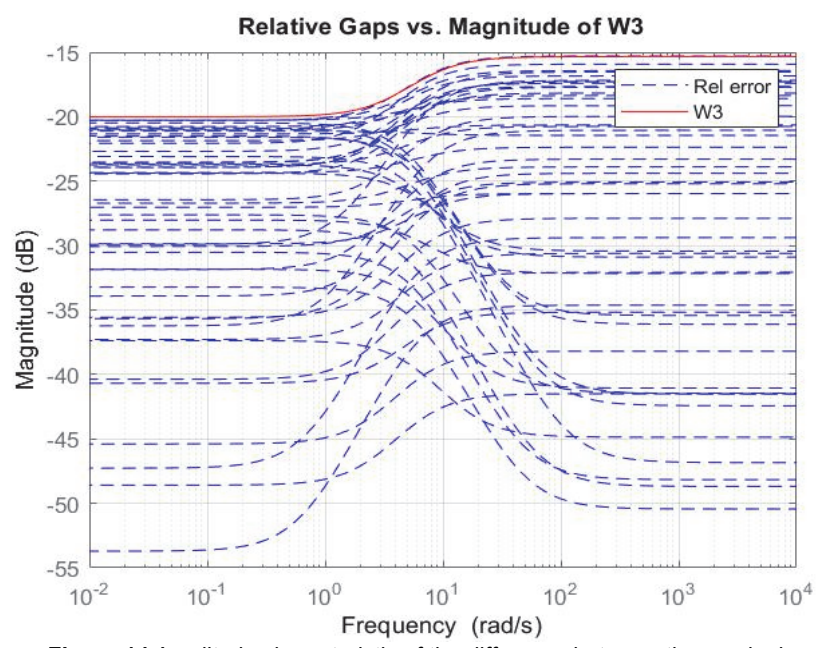

Figure 14 Amplitude characteristic of the difference between the nomina system and the family of perturbated systems

Finally, the robustness of the control circuit for the designed controller was verified graphically, according to the Fig. 3 describing the criterion of robust performance generally. Fig. 15 clearly indicates that the robust performance of the control has been fulfilled since there is no intersection of the circles.

However, it is important to notice that circles correspond to the filter, not only to certain value changes of a parameter itself, where the use of a polytop model may be used effectively. The circle represents a classical filer and in the form of $W_{3}$ it is easier for interpretation and for numerical computation.

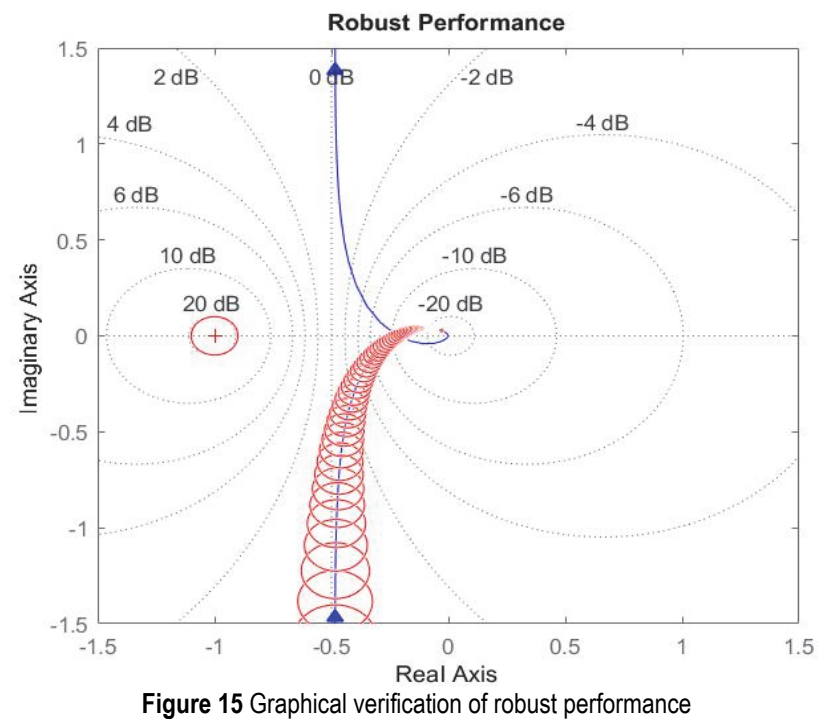

\section{CONCLUSION}

This paper demonstrated the mixed sensitivity problem briefly discussed in the introductory section with the use of an educational case study with alternative modifications. The effects of particular weighting functions on suboptimal controller design according to $H_{\infty}$-norm were investigated. The effect of filters $W_{1}$ and $W_{2}$ on the nominal system was demonstrated in the first part. Setting of both filters is very important to achieve the desired properties of the control circuit.

In the next part it was shown how to proceed with the design of the controller for the system with uncertainty, both in the gain and subsequently in both the gain and in the time constant of the system. The resulting designed control circuit was graphically verified, and the requirement of robust performance quality was met.

\section{Acknowledgment}

This work was supported by the European Regional Development Fund in the Research Centre of Advanced Mechatronic Systems project, project number CZ.02.1.01/0.0/0.0/16_019/0000867 within the Operational Programme Research, Development and Education.

This work was supported by the project SP2021/29, "Development of algorithms and systems for control, measurement and safety applications VII" of Student Grant System, VSB-TU Ostrava.

\section{REFERENCES}

[1] Gu, D. W., Petkov, P. H., \& Konstantinov, M. M. (2013). Robust Control Design with Matlab ${ }^{\circledR}$. London: Springer. https://doi.org/10.1007/978-1-4471-4682-7

[2] Ozana, S., Vojcinak, P., Pies, M., \& Hajovsky, R. (2013). Mixed Sensitivity H-Inf Control for Helicopter Model. IFAC Proceedings Volumes, 46(28), 104-109. https://doi.org/10.3182/20130925-3-CZ-3023.00076

[3] Lin, F. (2007). Robust control design: an optimal control approach, 18. Chichester, West Sussex, England: John Wiley/RSP. https://doi.org/10.1002/9780470059579

[4] Safonov, M. G. (2012). Origins of robust control: Early history and future speculations. Annual Reviews in Control, 36(2), 173-181. https://doi.org/10.1016/j.arcontrol.2012.09.001

[5] Skogestad, S. \& Postlethwaite, I. (2007). Multivariable feedback control: analysis and design, 2. New York: Wiley.

[6] Zhou, K., Doyle, J. C., \& Glover, K. (1996). Robust and optimal control, 40, 146. New Jersey: Prentice hall.

[7] Ortega, M. G. \& Rubio, F. R. (2004). Systematic design of weighting matrices for the $\mathrm{H} \infty$ mixed sensitivity problem. Journal of Process Control, 14(1), 89-98. https://doi.org/10.1016/S0959-1524(03)00035-0

[8] Postlethwaite, I., Tsai, M. C., \& Gu, D. W. (1990). Weighting function selection in $\mathrm{H} \infty$ design. IFAC Proceedings Volumes, 23(8), 127-132. https://doi.org/10.1016/S1474-6670(17)51904-7 


\section{Contact information:}

\section{Jakub NEMCIK, Ing}

(Corresponding author)

Department of Cybernetics and Biomedical Engineering

VSB-Technical University of Ostrava,

17. listopadu 2172/15, 70800 Ostrava, Czech Republic

E-mail: jakub.nemcik@vsb.cz

\section{Filip KRUPA, Ing.}

Department of Cybernetics and Biomedical Engineering

VSB-Technical University of Ostrava

17. listopadu 2172/15, 70800 Ostrava, Czech Republic

E-mail: filip.krupa@vsb.cz

Stepan OZANA, doc. Ing. PhD

Department of Cybernetics and Biomedical Engineering

VSB-Technical University of Ostrava,

17. listopadu 2172/15, 70800 Ostrava, Czech Republic

E-mail: stepan.ozana@vsb.cz

Lukas HUBKA, Ing. PhD

Department of Mechatronics and Technical Informatics,

Faculty of Mechatronics, Informatics and Interdisciplinary Studies,

Technical University of Liberec,

Studentska 1402/2, 46117 Liberec, Czech Republic

E-mail: lukas.hubka@tul.cz

Zdenek SLANINA, Ing. PhD

Department of Cybernetics and Biomedical Engineering,

VSB-Technical University of Ostrava,

17. listopadu 2172/15, 70800 Ostrava, Czech Republic

E-mail: zdenek.slanina@vsb.cz 